\title{
Energy Efficient Utilization of IEEE 802.11 Hot Spots in 3G Wireless Packet Data Networks
}

\author{
F. Ozan Akgül and M. Oğuz Sunay \\ Koç University, \\ Department of Electrical \& Electronics Engineering, \\ Rumeli Feneri Yolu Sariyer, Istanbul 34450, Turkey \\ fakgul@ku.edu.tr, osunay@ku.edu.tr \\ http://wireless.ku.edu.tr
}

\begin{abstract}
The third generation wireless networks and wireless local networks possess complementary characteristics. Recently, there has been significant interest in providing algorithms and specifications that enable their inter-operability. In this paper we propose a novel cross-network, cross-layer algorithm that jointly performs $3 \mathrm{G}$ resource allocation and adhoc mode WLAN routing towards effectively increasing the performance of the $3 \mathrm{G}$ system. The metrics used in this joint design ensures that multi-user diversity is exploited without causing user starvation in the $3 \mathrm{G}$ system and the WLAN assistance does not cause an unfair treatment to any of the mobiles from a battery usage point of view. Furthermore, the design attempts to select the WLAN route so that the assistance does not become a major part of the internal WLAN traffic.
\end{abstract}

\section{Introduction}

With the ever increasing use of the Internet there is a significant interest in making this technology available anywhere, anytime. To this end, the recent research in wireless systems has focused on providing high speed packet data access. Two complementary wireless technologies are gaining momentum to realize this goal: wireless local area networks (WLANs) providing high data rates over localized, small geographical areas (also referred to as hot spots), and thirdgeneration $(3 \mathrm{G})$ cellular systems designed to provide lower data rates over wide geographical areas.

Given the clear demand for both, and their complementary characteristics, there has been significant interest in providing specifications for the interoperability of $3 \mathrm{G}$ and WLAN systems. Both of the Third Generation Partnership Projects (3GPP and 3GPP2) have taken initiatives to develop cellular-WLAN interworking architectures that would be compatible to the existing $3 \mathrm{G}$ cellular system specifications of WCDMA and cdma2000 [1,2]. The goal is to provide cellular users seamless WLAN access where available and provide rates not achievable by $3 \mathrm{G}$ systems alone. Work on this front has focused on authentication, billing, seamless QoS and application-level services provisioning and roaming. 
Both 3GPP and 3GPP2 provide specifications for the cellular-WLAN interoperation when the WLAN system operates in the access network mode. However, the WLAN systems may be configured to operate in ad-hoc network mode as well. There has also been work in the literature that discuss the inter-operation of ad-hoc network mode WLAN with cellular systems [3-5]. In this case, the cellular system may be considered to be furnished with a relaying capability which would increase the overall coverage and capacity of the cellular system. This is because mobile terminals with channels experiencing low signal-to-noise ratios (SNRs) to the base station may observe better indirect, relayed links thereby increasing the observed data rate and/or reducing the power consumption. Clearly dual-network terminals would be necessary in this case as well.

In this paper we investigate the ad-hoc network mode WLAN and $3 \mathrm{G}$ wireless packet data integration where the cooperation would possibly enhance the cellular system capability. The $3 \mathrm{G}$ system under consideration is the North American IS-856 rev. 0 system (HDR) [6] which is a data-only cellular system providing peak rates of up to $2.4 \mathrm{Mbps}$ over a $1.25 \mathrm{MHz}$ bandwidth. Embedded in the area are hot spots (HSs) that are also covered with WLANs operating in the ad-hoc mode. The integration of the cellular and WLAN networks should enable the base station to know at any given time whether a dual-network mobile terminal is inside a given hot spot or not. Then, the cellular system will have the option of relaying information to the mobile terminal residing inside a hot spot via a number of other mobile terminals acting as relay nodes. For this scenario, we propose to jointly establish the cellular system resource allocation and the ad-hoc WLAN network routing schemes using a cross-network, cross-layer platform. While the IS-856 system will intend to increase the multi-user diversity gains through proper use of ad-hoc routing inside the hot spot, the routing algorithm will attempt to ensure that an energy fair route that utilizes as few of the WLAN resources as possible is set aside for this purpose.

The rest of the paper is organized as follows: next we give a brief overview of the $3 \mathrm{G}$ IS- 856 rev. 0 wireless packet data system and its resource allocation procedure. We then discuss the most common ad-hoc routing protocols that take battery use into account. We propose a new cross-network, cross-layer joint cellular system resource allocation and WLAN ad-hoc system routing algorithm for the coordinated $3 \mathrm{G}-\mathrm{WLAN}$ system. After briefly describing the simulation environment we present detailed simulation results for the proposed set-up and finally we present conclusions to the paper.

\section{Overview of the IS-856 System}

The wireless $3 \mathrm{G}$ system under consideration in this paper is the recently standardized 3G CDMA system for packet data, IS-856, otherwise known as HDR (High Data Rate) [6]. The HDR system divides the time into slots of length $1.67 \mathrm{~ms}$ and allocates all of its resources to a single user at a given time slot. Based on the observed channel quality between the base station and the served user at a given time, the modulation and coding levels are adjusted to provide 
the maximum possible transmission data rate. The information data is encoded in blocks called physical layer packets. For some of the data rates, the physical layer packets span multiple time slots. The available data rates in the IS-856 rev. 0 system are given in Table 1 along with the associated packet size, modulation and coding levels and the number of time slots necessary to transmit the physical layer packets.

Table 1. Available data rates in IS-856

\begin{tabular}{lllll}
\hline $\begin{array}{l}\text { Data Rate } \\
\text { (kbps) }\end{array}$ & Time Slots & $\begin{array}{l}\text { Packet Size } \\
(\text { bits })\end{array}$ & Code Rate & Modulation \\
\hline $38.4,76.8,153.6,307.2,614.4$ & $16,8,4,2,1$ & 1024 & $1 / 5$ & QPSK \\
$307.2,614.4,1228,8$ & $4,2,1$ & 2048 & $1 / 3$ & QPSK \\
$921.6,1843.2$ & 2,1 & 3072 & $1 / 3$ & $8-P S K$ \\
$1228.8,2457.6$ & 2,1 & 4096 & $1 / 3$ & $16-Q A M$ \\
\hline
\end{tabular}

At the heart of the IS-856 system there is a scheduler that selects which user to service at a given time instance. The choice of the scheduling algorithm affects the overall system throughput as well as the average delay experienced by users in between successive accesses to the system. The throughput-optimal scheduling rule is one where the user with the best channel conditions is scheduled for service for each time slot. In such a scenario, the larger the number of users in the system, the more likely it is to find a user experiencing a really good channel resulting in a better system throughput. This is referred to as multi-user diversity in the literature. The optimal scheduling algorithm would be impractical as users closer to the base station would almost always observe better channel conditions and thus would grab the system resources continuously. Then, ideally scheduling algorithms that provide fairness across subscribers while utilizing multi-user diversity as much as possible are desirable. The study of scheduling algorithms is an active research topic. In this paper we will utilize the exponential rule [7] since this rule has been shown to have a very good fairness-throughput trade-off performance.

To describe the exponential rule properly, let us first define the following: $t_{s}$ is the length of the time slot (=1.67 ms for the IS-856 system), $r_{i}\left(k t_{s}\right)$ is the data rate supported by user $i$ at the $k$ 'th time slot, $r_{i}\left(k t_{s}\right)$ is the average data rate observed by user $i$ defined over a long sliding window of length $T$ slots spanning the time $\left[(k-1-T) t_{s},(k-1) t_{s}\right], l_{i}\left(k t_{s}\right)$ is the number of slots user $i$ has spent without service and $l\left(k t_{s}\right)$ is the average of latencies observed by all users up until time slot $k$. Then, for the $k$ 'th time slot the exponential rule selects user $j$ such that

$$
j=\arg \max \frac{r_{i}\left(k t_{s}\right)}{r_{i}\left(k t_{s}\right)} \exp \left(\frac{l_{i}\left(k t_{s}\right)-\overline{l\left(k t_{s}\right)}}{1+\sqrt{\overline{l\left(k t_{s}\right)}}}\right)
$$


In (11), a large latency observed by one of the users relative to the overall average latency results in a very large exponent, overriding the channel conditions and leading to the large latency user getting priority. On the other hand, for small weighted latency differences, the exponential term is close to 1 and the policy is only ruled by the users' channel conditions relative to their own means.

\section{Energy-Aware Multi-hop Routing in the WLAN Network}

Lacking network infrastructure, wireless ad-hoc networks have no routers planning or overseeing the data transmission between two nodes. Instead, the nodes themselves function as routers and they discover and maintain routes to other nodes in the network. Several routing protocols have been proposed in the literature for ad-hoc networks. In general, these protocols can be divided into two broad classes: table-driven, or on-demand routing protocols.

Table-driven protocols require each node to maintain an accurate routing table in which information regarding every possible destination node is maintained at all times. The changes in the network topology is handled by sending update broadcast messages. Several table-driven protocols have appeared in the literature. These vary in the information stored at the routing table and how information updates are handled. The on-demand routing protocols, on the other hand, create routes only when requested by a source node. Upon demand, the source node initiates a route discovery process which lasts until a route is found or all possibilities are exhausted. Once a route between two nodes is established, this route is maintained by a route maintenance procedure until it is no longer desired or until the destination node becomes inaccessible by any means.

In wireless ad-hoc networks most nodes will operate on batteries. For this reason, minimization of the overall battery consumption and fair distribution of this consumption across the nodes become an important issue. By incorporating the current power levels into the routing tables in table-driven protocols and in the route discovery messages in the on-demand protocols, a route selection based on power consumption is possible. Such protocols should aim to select the path that minimizes the total power needed for the transmission between the source and destination nodes while minimizing the power consumption of all of the nodes. A number of power-efficient routing protocols have been studied in the literature for wireless ad-hoc networks that achieve one or both of these goals. The Maximum Total Transmission Power Routing Protocol (MTTPR), aims to find the route where the total transmission power used is a minimum [8]. MTTPR usually selects routes with more hops than other algorithms. This may not be desirable since a route with multiple routes will cause greater endto-end delay. Perhaps the biggest disadvantage of the MTTPR protocol is that while it aims to select the route with the smallest overall transmission power, it pays no regards to the individual power levels of the nodes. The Minimum Battery Cost Routing (MBCR) aims to correct this by associating a cost function with every node that is inversely proportional with its remaining battery level 
[9]. The protocol aims to select the route with the minimum total cost function. While the protocol incorporates fairness in the battery usage across the network, it does not prevent selecting a route that includes a node with a critically low power level. The Min-Max Battery Cost Routing Protocol (MMBCR) aims to minimize the maximum battery cost function within the route [9]. While this protocol ensures that no critical node appears in the final route, it does not minimize the overall power consumption. An alternative, the Conditional MinMax Battery Cost Routing Protocol (MMBCR) aims to choose the route with the minimum overall power consumption among the routes that all have the maximum battery cost function below a certain threshold [10].

\section{Proposed 3G-WLAN Integration}

In this paper we propose the inter-operation of $3 \mathrm{G}$ and WLAN networks when both networks are available on the same geographical area. Our goal is to enhance the multi-user diversity impact observed in the IS-856 system by allowing multi-hop routing within the hot spot as an intermediate step on route to the destination mobile terminal. Then, the IS-856 base station, employing the exponential scheduler of (1) will need to check after each scheduling decision whether the scheduled mobile terminal is a dual-network, active WLAN subscriber as well. In a tightly-coupled integration scenario, the registration information of the two networks can easily be shared. In a loosely coupled integration scenario, the mobile terminals can transmit back to the base station occasional messages regarding whether they are also an active WLAN user or not. This information could be integrated into the regular channel state feedback messages the terminals transmit every $1.67 \mathrm{~ms}$ in the IS-856 system.

If the scheduled user is not an active WLAN user, the service to that user will be completed within the 3G network without any changes to the IS-856 operation. If, on the other hand, the mobile is also a WLAN subscriber, the base station will need to modify the scheduling decision to select a WLAN user that will potentially act as a gateway on route to the initially scheduled mobile. The scheduling of the gateway mobile should be such that the wireless channel it experiences from the $3 \mathrm{G}$ base station is better than the scheduled destination mobile terminal.

Then, user $k$ is scheduled to act as the gateway mobile when

$$
k=\arg \max _{i \in A} r_{i}\left(k t_{s}\right)
$$

where maximization is done across all mobile terminals that are currently in the hot spot and that are dual network capable. This set of terminals is denoted as the set $A$.

Once the potential gateway terminal is determined, the $3 \mathrm{G}$ base station will need to compare the achievable data rates of this mobile with that of the destination mobile. If the destination mobile has a larger or equal data rate than the selected gateway mobile, then it can be serviced directly without the need for WLAN assistance. If, on the other hand, the gateway mobile has a larger data 
rate than the destination mobile, WLAN assistance is helpful. In this case, the destination mobile can be serviced at the gateway mobile data rate (assuming that a route can always be found within the WLAN so that the bottleneck for the data rate is always the wireless channel from the base station to the gateway mobile).

If multiple terminals result in the same, maximum data rate, ties may possibly be broken in such a way that the node with the highest battery capacity is chosen. Naturally, for this purpose, the $3 \mathrm{G}$ network base station needs to know the current battery levels of all dual-network terminals that are active WLAN users. It is possible to incorporate this information into the channel-state feedback the terminals regularly transmit in the IS-856 network.

After the selection of the gateway terminal, a route between this terminal and the destination terminal needs to be discovered. The route discovery may be handled within the WLAN network without any input from the $3 \mathrm{G}$ network. In fact, the $3 \mathrm{G}$ network does not need to know the route details at all. In the 3G-WLAN cooperation scenario, a number of factors need be taken into account when deciding on the multi-hop route. First, the use of the WLAN system towards bettering the $3 \mathrm{G}$ system performance should result in as little impact on the internal WLAN traffic flow as possible. End-to-end delay between the gateway mobile and the destination user should also be accounted for by selecting a route that offers a relatively fast transmission speed. Second, the routing protocol has to ensure that as little power as possible is used for this purpose. Furthermore, it has to make sure that the power drainage should be shared more or less equally by all of the WLAN users so that some level of fairness can be maintained. None of the power-aware routing schemes described in the literature provide a solution for this problem since none consider battery consumption and transmission rate simultaneously. In this paper we propose an Power-Aware Exponential Ad-Hoc Routing Protocol (PEAR). PEAR selects the route $r$ between the gateway terminal and the destination terminal such that

$$
r=\arg \max _{i \in B} R_{i}(t) \exp \left(\frac{c_{i}(t)-\overline{c(t)}}{1+\sqrt{\overline{c(t)}}}\right)
$$

where $R_{i}(t)$ is the normalized average throughput and $c_{i}(t)$ is the sum percentile battery capacities of the nodes for route $i$ at time $t$, respectively. $\overline{c(t)}$ is the average of the sum percentile battery capacities of all possible routes. The set $B$ is the set of routes where the minimum battery capacity of a node is above a given threshold. The normalized throughput for route $i$ with $D_{i}$ nodes and data rates of $R_{\left(n_{i}, n_{i-1}\right)}(t)$ between nodes $n_{i}$ and $n_{i-1}$ can be calculated as,

$$
R_{i}(t)=\frac{1}{D_{i}-1} \sum_{k=1}^{D_{i}-1} \frac{R_{\left(n_{i}, n_{i+1}\right)}(t)}{R_{\max }}
$$

where $R_{\max }$ is the maximum possible transmission rate in the WLAN system (e.g. 11 Mbps for IEEE 802.11b). The sum percentile battery capacity for route 
$i$ with $D_{i}$ nodes each with current percentile battery capacities $c_{\left(n_{i}\right)}(t)$ is calculated as,

$$
c_{i}(t)=\sum_{k=1}^{D_{i}} c_{\left(n_{i}\right)}(t) .
$$

Similarly, the average of the sum percentile battery capacities of all possible $N$ routes is calculated as,

$$
\overline{c(t)}=\frac{1}{N} \sum_{i=1}^{N} c_{i}(t) .
$$

From (3), it is clear that for routes that have a large sum percentile battery, the exponential term dominates the data rate term and thus favoring routes with greater battery capacities. This leads to a fairer distribution of battery consumption across the WLAN user population. The data rate term in (3) is also an important parameter in the route selection since it directly relates to the link utilization of a particular route within the hot spot. Relaying the packets to the destination node on a route that has the highest average throughput means the minimal disruption of the internal WLAN communications. Thus, the proposed routing protocol, PEAR provides a compromise between choosing the link with the largest average data rate and the route with the largest total battery capacity. When the total battery capacity of a certain route is greater than the average total batter capacity of all of the routes by more than order $\sqrt{\overline{c(t)}}$, the exponential term overrides the average route throughput and this route is more likely to be selected. For routes with lower battery capacities than the average total battery capacity of all routes, the argument is dominated by the average throughput term. When a certain route has significantly lower battery capacity, this route will be less likely to be chosen even if its average bit rate is high.

\section{System Performance Evaluation}

To assess the performance of the WLAN assisted 3G IS-856 system described above, we have performed detailed simulations. The simulations are composed of three stages: System Level Simulations, Physical Layer Simulations and Joint Resource Allocation and Routing Simulations.

In the system level simulations we consider a 2-tier 19-cell environment for the $3 \mathrm{G}$ IS-856 system. Here, the first tier has 6 and the second tier has 12 cells centered around the cell of interest. Each cell is considered to have a radius of 1 $\mathrm{km}$ in the layout. We consider various hot spot configurations within the center cell. HSs that are $250 \mathrm{~m}, 500 \mathrm{~m}$ and $750 \mathrm{~m}$ away from the $3 \mathrm{G}$ base station are considered. The HSs are assumed to have radii of $100 \mathrm{~m}$ or $200 \mathrm{~m}$. We assume that the $3 \mathrm{G}$ users that are outside the hot spot have velocities of $3 \mathrm{~km} / \mathrm{h}$ and $3 \mathrm{G}$ users that have WLAN access inside the HSs are assumed to have velocities of 0.5 $\mathrm{km} / \mathrm{h}$. The channel model in both cases includes path loss, Rayleigh fading and shadow fading. The ITU Pedestrian-A model describes the path loss model [11]. 
Similarly, Gudmundson's shadow fading model is used to describe the process as a log-normal random process [12]. The small-scale Rayleigh fading has been modeled using a filtered Gaussian noise. The sampling rate for the simulations is $600 \mathrm{~Hz}$. The simulations have been performed for 18000 slots corresponding to 30 seconds of real time.

We have then performed comprehensive physical layer simulations to find the $\operatorname{SNR}\left(E_{c} / I_{0}\right)$ values corresponding to the target $1 \%$ packet error probabilities for each of the system data rates listed in Table 1. Agilent's ADS 2005A package has been used to simulate the IS-856 air interface components. The minimum required $E_{c} / I_{0}$ values to support each of the data rates is given in Table 3 .

Table 2. Minimum Required $E_{c} / I_{0}$ Levels for $1 \%$ Packet Error Rate

\begin{tabular}{|c|c|c|c|c|c|}
\hline Rate (kbps) & Slot & $E_{c} / I_{0}(\mathrm{~dB})$ & Rate (kbps) & Slots & $E_{c} / I_{0}(\mathrm{~dB})$ \\
\hline 38.4 & 16 & -11.68 & 614.4 & 2 & -0.88 \\
\hline 76.8 & 8 & -9.31 & 1228.8 & 1 & 3.55 \\
\hline 153.6 & 4 & -6.14 & 921.6 & 2 & 1.58 \\
\hline 307.2 & 2 & -2.96 & 1843.2 & 1 & 7.73 \\
\hline 614.4 & 1 & -0.77 & 1228.8 & 2 & 3.62 \\
\hline 307.2 & 4 & -3.94 & 2457.6 & 1 & 11.19 \\
\hline
\end{tabular}

Finally, we have performed the joint resource allocation and routing simulations for a total number of $N_{3 G}=323 \mathrm{G}$ users such that

$$
N_{3 G}=N_{H S}+N_{H S}^{\prime}
$$

where $N_{H S}$ is the number of $3 \mathrm{G}$ users that are also in the WLAN hot spot and $N_{H S}^{\prime}$ is the number of $3 \mathrm{G}$ users outside the WLAN coverage. In this scenario, we investigate the performance of the joint resource allocation and ad-hoc routing scheme for 2 to 16 active users in the hot spot, corresponding to a penetration of $6 \%-50 \%$.

For each user, the current supported data rate along with the battery level is assumed to be fed back to the base station for scheduling and, if necessary, gateway terminal selection. The feedback messages from the terminals are assumed to arrive at the base station error-free, but with a round-trip delay of 3 time slots. The base station keeps a track of the average observed data rates for each active user where the averages are computed using a sliding window of 1000 slots.

Once the scheduling and gateway terminal selection is performed, the ad-hoc route is determined using (3). It is assumed that routing is performed locally within the WLAN at the initiation of the gateway terminal and the $3 \mathrm{G}$ network need not keep track of the details. In practice, the WLAN assistance will provide throughput gains at the expense of a certain delay due to route discovery, and multi-hop transmission within the network. Such additional delay is not considered in the scheduling process. In fact, the scheduling algorithm of (1) is 
assumed to consider only the delay due to resource allocation procedure of the $3 \mathrm{G}$ system.

The route selection requires the knowledge of the battery levels of the nodes as well as the data rates between the nodes. We assume that the WLAN system under consideration is the IEEE 802.11 b system for which the data rate-range relationship is such that [13] data rates of $11 \mathrm{MBps}$ is achievable for open field ranges of $160 \mathrm{~m}$ and smaller, $5.5 \mathrm{Mbps}$ is achievable for ranges of $160 \mathrm{~m}-270 \mathrm{~m}, 2$ Mbps is achievable for ranges of $270 \mathrm{~m}-400 \mathrm{~m}$, and finally $1 \mathrm{Mbps}$ is achievable for ranges of $400 \mathrm{~m}-550 \mathrm{~m}$. No WLAN link is available between mobiles that are at least $550 \mathrm{~m}$ apart.

The battery consumption inside the WLAN system is modeled such that each packet transmission or reception consumes one unit from battery. For instance the source and the destination nodes will consume 1 unit of battery whereas all intermediate nodes on the route will consume 2 units since they both receive and transmit the packets.

Based on the data rates and battery levels of each node, all possible routes are compared using the metric given in (3) and the route that provides the maximum value is selected. Figure 1 shows the WLAN assisted $3 \mathrm{G}$ performance results along with the performance of the $3 \mathrm{G}$ system without the WLAN assistance in the same cell geometry. It is observed that as the number of users in the hot spot increases, the assistance of the WLAN becomes more useful. This is to be expected, as the number of users inside the hot spot increase, it becomes more likely to find a WLAN gateway mobile experiencing a channel much better than that of the original scheduled user. In other words, as the number of users in the WLAN increase, the WLAN assistance enhances the multi-user diversity gain even when the scheduler tries to be fair across the $3 \mathrm{G}$ users.

It is also observed that as the WLAN hot spot location moves away from the cell radius, higher gains are observed through WLAN assistance. For WLAN HSs close to the cellular radius, most of the users within the hot spot will observe good channel conditions. The likelihood of finding a user that has better channel conditions than that of the originally scheduled user will be smaller in this case. Indeed, when one looks at the percentage of times WLAN assistance is beneficial, it becomes clear that it is less likely to gain from WLAN assistance if the hot spot is closer to the cell center. Alternatively, for HSs that are closer to the cell boundaries, larger variations across users in terms of achievable data rates are observed. In this case, WLAN assistance achieves gains most of the times.

In Figure 1 the $3 \mathrm{G}$ system throughput sometimes increases and sometimes decreases as the WLAN penetration increases for both the $3 \mathrm{G}$ only and WLAN assisted system scenarios. The dual nature is completely due to the geometry of the cell in question. Recall that the simulations are performed keeping the total number of users in the cell fixed at 32 . As more users are forced into the hot spot, the geometry moves further away from a uniform distribution of these 32 mobiles in the cell. If the WLAN hot spot is closer to the cell center, clearly more and more users will observe better channel characteristics and thus the overall system improvement will increase with increased concentration in the WLAN hot spot. 

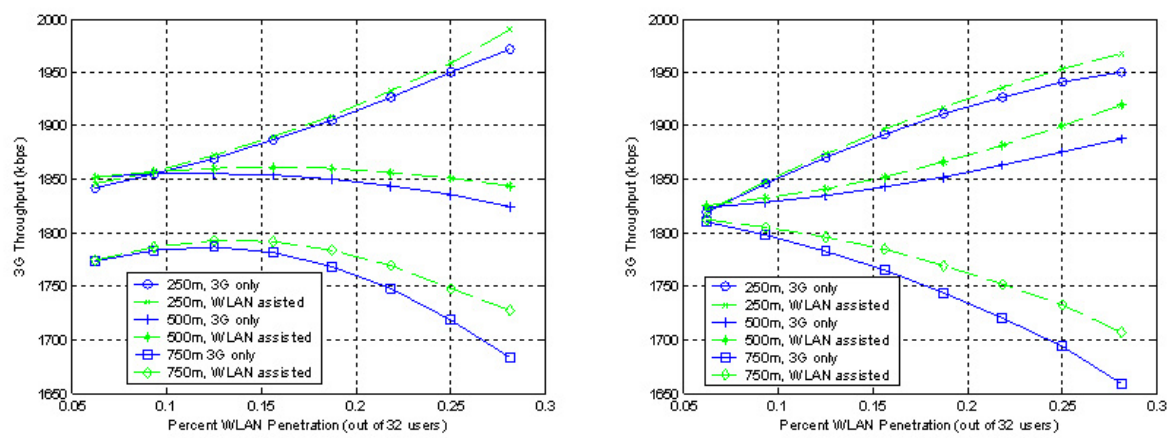

Fig. 1. Impact of the WLAN Assistance on the 3G system performance when the WLAN radius is $100 \mathrm{~m}$ and $200 \mathrm{~m}$, respectively
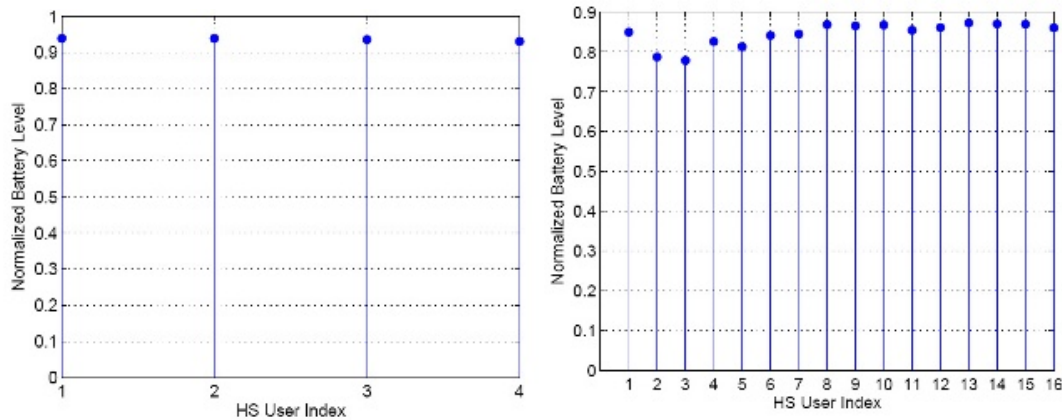

Fig. 2. Final Battery Levels of the Terminals (i. 4 of the 32 users are in the hot spot of radius $100 \mathrm{~m}$ that is $500 \mathrm{~m}$ away from the BS, ii. 16 of the 32 users are in the hot spot of radius $100 \mathrm{~m}$ that is $500 \mathrm{~m}$ away from the BS )

If on the other hand, the hot spot is closer to the cell boundary, increasing the WLAN user concentration will force more users to be located away from the cell center, decreasing the overall system throughput as the concentration increases.

Figure 2 shows the battery levels for selected number of users $(4,16$ HS users, respectively) to demonstrate the fair selection behavior of our proposed scheme. The battery levels are the normalized values with respect to the full battery level. The graphs given here are for a WLAN hot spot that is $500 \mathrm{~m}$ from the $3 \mathrm{G}$ base station and has $100 \mathrm{~m}$ radius. Very similar graphs are obtained for hot spots that are 250 and 750 meters away from the $3 \mathrm{G}$ base station. Furthermore, the coverage area of the WLAN hot spot does not seem to affect the battery consumption fairness of the algorithm.

One important result we have obtained through the simulations is that the maximum number of hops in the selected route saturate at 3 . This means that by 
Table 3. Hop Percentages as a Function of HS User Concentration and HS Topology

\begin{tabular}{|c|c|c|c|c|c|c|c|c|}
\hline \multicolumn{9}{|c|}{ 100m Radius Hot Spot } \\
\hline \multicolumn{3}{|c|}{$250 \mathrm{~m}$ away from BS } & \multicolumn{3}{|c|}{$500 \mathrm{~m}$ away from BS } & \multicolumn{3}{|c|}{$750 \mathrm{~m}$ away from BS } \\
\hline No Users & Hops & $\%$ & No Users & Hops & $\%$ & No Users & Hops & $\%$ \\
\hline \multirow[t]{5}{*}{4} & \multicolumn{2}{|c|}{$3 \mathrm{G}$ only 89.172} & 4 & \multicolumn{2}{|c|}{$3 \mathrm{G}$ only 89.217} & 4 & \multicolumn{2}{|c|}{$3 \mathrm{G}$ only 86.828} \\
\hline & 0 & 2.6389 & & 0 & 2.9889 & & 0 & 3.4389 \\
\hline & 1 & 7.4722 & & 1 & 8.2444 & & 1 & 9.5333 \\
\hline & 2 & 0.7167 & & 2 & 0.4444 & & 2 & 0.1333 \\
\hline & 3 & 0 & & 3 & 0.1056 & & 3 & 0.0667 \\
\hline \multirow[t]{5}{*}{$\overline{16}$} & \multicolumn{2}{|c|}{$3 \mathrm{G}$ only 59.322} & \multirow[t]{5}{*}{16} & \multicolumn{2}{|c|}{$3 \mathrm{G}$ only 59.278} & \multirow[t]{5}{*}{16} & \multicolumn{2}{|c|}{$3 \mathrm{G}$ only 56.267} \\
\hline & 0 & 2.5833 & & 0 & 2.5444 & & 0 & 2.6944 \\
\hline & 1 & 35.972 & & 1 & 36.306 & & 1 & 38.539 \\
\hline & 2 & 2.1222 & & 2 & 1.8722 & & 2 & 2.5 \\
\hline & 3 & 0 & & 3 & 0 & & 3 & 0 \\
\hline \multicolumn{9}{|c|}{ 200m Radius Hot Spot } \\
\hline \multicolumn{3}{|c|}{$250 \mathrm{~m}$ away from BS } & \multicolumn{3}{|c|}{$500 \mathrm{~m}$ away from BS } & \multicolumn{3}{|c|}{$750 \mathrm{~m}$ away from BS } \\
\hline No Users & Hops & $\%$ & No Users & Hops & $\%$ & No Users & Hops & $\%$ \\
\hline \multirow[t]{5}{*}{$\overline{4}$} & $3 \mathrm{G}$ only & 89.956 & \multirow[t]{5}{*}{4} & $3 \mathrm{G}$ only & 89.05 & \multirow[t]{5}{*}{4} & \multicolumn{2}{|c|}{$3 \mathrm{G}$ only 87.683} \\
\hline & 0 & 2.6333 & & 0 & 2.7333 & & 0 & 2.9389 \\
\hline & 1 & 4.0167 & & 1 & 3.7 & & 1 & 4.4333 \\
\hline & 2 & 2.8667 & & 2 & 2.7944 & & 2 & 2.6944 \\
\hline & 3 & 1.5278 & & 3 & 1.7222 & & 3 & 2.25 \\
\hline \multirow[t]{5}{*}{$\overline{16}$} & 3G only & 58.967 & \multirow[t]{5}{*}{16} & 3G only & 58.711 & \multirow[t]{5}{*}{16} & \multicolumn{2}{|c|}{$3 \mathrm{G}$ only 56.656} \\
\hline & 0 & 2.3444 & & 0 & 2.6389 & & 0 & 2.5278 \\
\hline & 1 & 16.522 & & 1 & 16.561 & & 1 & 16.1 \\
\hline & 2 & 13.2 & & 2 & 13.639 & & 2 & 15.328 \\
\hline & 3 & 8.9667 & & 3 & 8.45 & & 3 & 9.3889 \\
\hline
\end{tabular}

utilizing at most 2 intermediate nodes, the $3 \mathrm{G}$ packet is relayed to the destination. Table 3 provides the detailed results of percentages that a certain number of hops occur for the topologies as well as user concentrations considered.

One immediate result obtained is the percent increase in the occurrence of higher number of hops as the number of users in the hot spot increases. This result is expected since as the number of users increase so do the number of possible routes requiring more nodes. However, despite this tendency, the results clearly indicate that the proposed algorithm favors fewer hops. It is also observed once again that WLAN assistance is more useful as the number of WLAN users increase. Another observation is that as the coverage area of the WLAN increases so do the expected number of hops. The reason for this is that, with increased WLAN coverage, it is more likely that the distance between the gateway terminal and the destination terminal is far, requiring multiple nodes for relaying along the way. As the number of users further increase in this case, the expected number of hops further increase. This is because, given equal distribution of battery levels, the algorithm will favor links with larger average data rates, which would most likely result in multiple hops where each node is separated by a shorter distance. 


\section{Conclusions}

In this paper we have investigated a possible system integration between the 3G IS-856 packet data system and the IEEE 802.11 WLAN operating in the ad-hoc network mode. We have proposed a cross-network, cross-layer approach to jointly determine how the $3 \mathrm{G}$ system resources are scheduled across the users so that a compromise is maintained between high network throughput and fairness across the user observed latency. The proposed scheme exploits multi-user diversity in the $3 \mathrm{G}$ system and employs a novel power-aware routing protocol, PEAR, that aims to find a compromise between selecting a route with high data rate and a route with large available battery capacity. Simulation results show improvements in the throughput performance of the $3 \mathrm{G}$ system when WLAN assistance is utilized. The proposed routing algorithm provides a fair use of battery capacity across the WLAN membership.

\section{References}

1. 3GPP, "Group Services and System Aspects: 3GPP Systems to Wireless Local Area Network (WLAN) Interworking - System Description (Release 6)," TS23.234. v.2.5.0, March 2004 .

2. 3GPP2, "3GPP2-WLAN Interworking, Stage 1 Requirements," SP0087-0 v.0.5, 14 July 2003.

3. V. Sreng, H. Yanikomeroglu and D.D. Falconer, "Relayer Selection Strategies in Cellular Networks with Per-to-Peer Relaying," Proceedings of the IEEE VTC-Fall 2003 Conference, 2003.

4. H. Wu et.al., "Integrated Cellular and Ad-Hoc Relaying Systems: iCAR," IEEE Journal on Selected Areas in Communications, vol. 19, pp. 2105-2115, 2001.

5. H.Y. Wei, R.D. Gitlin, "Two-Hop-Relay Architecture for Next-Generation WWAN/WLAN Integration," IEEE Wireless Communications Magazine, vol. 11, no.2, pp. 24-30, February 2004.

6. TIA/EIA/IS-856, "cdma2000 High Rate Packet Data Air Interface Specification," 3GPP2, C.S0024, v4.0, Oct. 2002.

7. S. Shakkottai, A. Stolyar, "Scheduling Algorithms for a Mixture of Real-Time and Non-Real-Time Data in HDR", Bell Laboratories Technical Report, 2000.

8. S. Singh, C.S. Raghavendra, "PAMAS - Power Aware Multi-Access Protocol with Signaling for Ad-Hoc Networks", ACM Computer Communication Review, vol.28, no.3, pp 5-26, July 1998.

9. S. Singh, M. Woo, and C.S. Raghavendra "Power Aware Routing in Mobile Ad-Hoc Networks", Mobile Computing and Networking, pp. 181-190, 1998.

10. C-K. Toh, "Maximum Battery Life Routing to Support Ubiquitous Mobile Computing in Wireless Ad-Hoc Networks," IEEE Communications Magazine, no.6, pp. 138-147, June 2001.

11. International Telecommunication Union, "Guidelines for Evaluation of Radio Transmission Technologies for IMT-2000," Recommendation, ITU-R, M.1225, 1997.

12. M. Gudmundson, "Correlation Model for Shadow Fading in Mobile Radio Systems," Electronics Letters, vol. 27, pp. 2145-2146, Nov. 1991.

13. Agere Systems, "WaveLAN 802.11b Chip Set for Standard Form Factors", Preliminary Product Brief, December 2002. 